\title{
Outcome of Delayed Decompression Surgery for Cauda Equina Syndrome secondary to Lumbar Disc Herniation: A Case Report
}

\author{
Lim SMa, Johari $J^{a}$, Yusof $M^{a}{ }^{a}$ \\ ${ }^{a}$ Department of Orthopaedics, Universiti Sains Malaysia, Kubang Kerian, Kelantan, Malaysia.
}

\section{ABSTRACT}

Cauda equina syndrome (CES) is a constellation of symptoms which consist of low back pain, sciatica, saddlearea paraesthesia, urinary or faecal incontinence, with or without motor weakness, and sensory deficit.

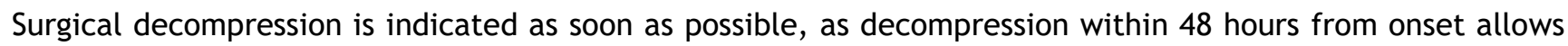
maximum improvement of symptoms. Recovery usually occurs months or years postoperatively. We report a case of a patient with cauda equina syndrome secondary to massive lumbar disc herniation who had undergone urgent decompression one week after onset of urinary and bowel dysfunction. The clinical outcome post surgery was also discussed.

KEYWORDS: cauda equina syndrome, herniated lumbar disc, delayed decompression INTRODUCTION

Cauda equina syndrome (CES) is a rare but potentially disabling neurologic affliction that occurs due to compression of lumbosacral nerve roots. Patients may present with bilateral leg pain and weakness, saddle anaesthesia and bladder/bowel dysfunction. Considered a surgical emergency, surgical decompression within 48 hours of the onset of symptoms had been deemed the consensus for maximum improvement of clinical signs and symptoms.

\section{CASE REPORT}

A 30-year-old male presented with low back pain and bilateral lower limb numbness for one week, associated with reduced sensation over the perianal region. He had also been having difficulty in passing urine and constipation for the past one week. There was no lower limb weakness. He had been experiencing bilateral lower limb numbness for four months. He could still ambulate till one week ago, when severe back pain rendered him bedridden.

Dr. Lim Soo May

Department of Orthopaedics

Hospital Universiti Sains Malaysia.

Jalan Raja Perempuan Zainab 2, Kubang Kerian, 16150 Kota Bharu, Kelantan, Malaysia

Tel No : +6012 4736125

Email: mayx1012@gmail.com
There was no history of trauma or heavy lifting. However, as a lorry driver, he sits for long hours when driving.

Upon examination, there was no spinal tenderness. Sensation to light touch and pin prick over L4 downwards was reduced, power over lower limb was full. Ankle reflex over bilateral ankles were diminished. Straight leg raising test was positive bilaterally at 60 degrees. There was reduced perianal sensation and deep anal sensation, anal tone was lax.

An urgent MRI revealed a large central L5S1 disc protrusion was compressing on the thecal sac and spinal nerves (Figure 1\&2). Patient had undergone open decompression the day after admission. Intraoperatively, a right-sided massive L5S1 disc was noted to be compressing the exiting $L 5$ nerve roots, traversing S1 nerve roots and central cauda equine nerve roots. Epidural tissue was oedematous and bulging. Bilateral L5S1 partial laminectomy was done.

Immediately postoperatively, numbness over the lower limbs started reducing. On day 2 postoperatively, perianal and deep anal sensations were normal, anal tone was intact, and voluntary anal contraction was present. On postoperative day 5 , after continuous bladder drainage removal, the patient was able to pass urine. At 3-week follow up, the patient was pain-free and able to ambulate. The symptoms were completely resolved. There was no 


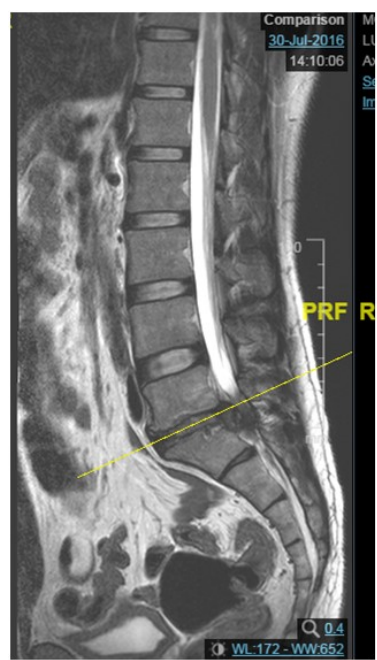

Figure 1: T2-weighted MRI mid sagittal cut indicating caudad migration of protruded L 5S1

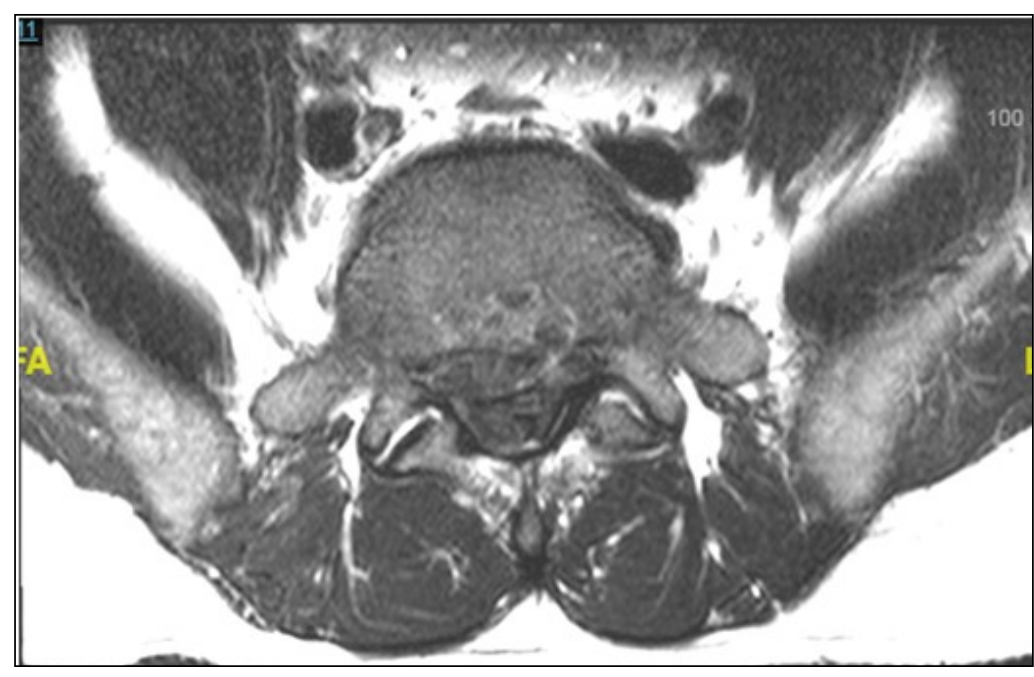

Figure 2: T2-weighted MRI axial cut at level of L581 disc indicating a massive central disc protrusion almost completely obliterating the spinal canal urinary/bowel dysfunction or perianal numbness. There was only residual sensory loss over right S1 region. At 7 weeks follow-up, he remained symptom free, and there was no neurological deficit.

\section{DISCUSSION}

The most caudal end of the spinal cord or "conus medullaris" is located at the level of the L1L2 intervertebral disc. Lumbosacral nerve roots or "cauda equine" that emerge from conus medullaris provide motor and sensory innervation to the lower extremities, pelvic floor, and bowel/bladder sphincters; in CES, the nerves supplying these regions are dysfunctional.

One of the most common causes of CES is a massive prolapsed lumbar intervertebral disc. Of these, $70 \%$ had a history of chronic low back pain; and in 30\% CES was the first symptom of disc herniation. A high index of suspicion has to be maintained, particularly when patients with prolapsed disc complain of urinary or bowel retention/incontinence. Urinary dysfunction and "saddle anaesthesia" are the most consistent identifying features. ${ }^{1}$ Other symptoms include lower limb radicular pain, weakness and numbness, with or without low back pain.

CES is generally treated as a surgical emergency, the diagnosis conventionally made by MRI. However, the role of urgent surgery in improving the outcome of CES secondary disc prolapse remains controversial. Some studies showed improved outcome from emergency decompression while others showed no benefit. Earlier studies advocated that surgical treatment be performed within 6 hours after onset of acute CES. More recent studies by Ahn and Shapiro revealed that the outcome of decompression surgery done within 48 hours was much better compared to that done after 48 hours. ${ }^{2,3}$

Yet other authors like Qureshi stated that emergency decompressive surgery did not significantly improve outcome in CES compared with a delayed approach, ${ }^{4}$ and may even cause more harm when performed under less than optimal conditions in the emergency setting. Several authors including McCarthy found that there was no correlation between the duration of onset of symptoms and surgery, and the extent of recovery. ${ }^{5}$ However, early surgery is still recommended to prevent an incomplete CES from progressing to complete one, with total loss of sphincter, motor, and sensory function.

In developing countries, disc herniation presenting as CES is more common due to factors like poverty, ignorance, limited availability of spine surgeons, and apprehension to seek surgical treatment. ${ }^{1}$ Our case report centers on a patient who had presented relatively late, i.e. one week after onset of low back pain, numbness of lower limbs and around the gluteal region, difficulty in urination and constipation. Lower limb numbness had begun four months prior to the presentation. In Shapiro's report, in the presentation of CES, $70 \%$ of patients presented with bilateral sciatica as the initial symptom indicating impending compression of the lumbosacral nerve roots. Another observation was that urinary retention always preceded urinary incontinence. $^{3}$ Our patient had undergone 
decompressive surgery one week after the onset of urinary/bowel dysfunction. However, he had regained normal bowel/bladder functions within 3 weeks post-operatively, and complete recovery of sensation over lower limbs in 7 weeks. A study by Dhatt suggested that there was definite benefit of decompression in patients operated at a mean delay of 12 days after onset of symptoms, but recovery time will be significantly delayed. ${ }^{1}$ The outcome of our patient is also in accordance to the report by Aly et al., that I,s of 14 patients with cauda equina syndrome who had undergone decompression 1 to 3 months after onset of sphincter disturbance had attained complete recovery postoperatively. ${ }^{6}$

\section{CONCLUSION}

CES secondary to a massive lumbar disc herniation is a rare but serious condition which diagnosis must not be missed, and be operated on as soon as possible. Decompression performed within 48 hours from onset of symptoms offers a maximum improvement of symptoms. However, our case report serves to show that delayed surgical decompression offers a good outcome with total resolution of symptoms. Thus, it has to be emphasized that even though patients present after the 48 hours golden period, they should not be treated as a semi-emergency case or planned for the next available elective operation time. Instead, surgical decompression has to be done with urgency, as the sooner the compressed nerve roots are released, the more likely it is that the patient will not be left with residual neurological deficits.

\section{REFERENCES}

1. Dhatt S, Tahasildar N, Tripathy SK, Bahadur R, Dhillon M. Outcome of spinal decompression in Cauda Equina syndrome presenting late in developing countries: case series of 50 cases. Eur Spine J Off Publ Eur Spine Soc Eur Spinal Deform Soc Eur Sect Cerv Spine Res Soc. 2011;20 (12):2235-2239.

2. Ahn UM, Ahn NU, Buchowski JM, et al. (2000) Cauda Equina syndrome secondary to lumbar disc herniation: a meta-analysis of surgical outcomes. Spine 25:348-352

3. Shapiro S. Cauda Equina syndrome secondary to disc herniation. Neurosurgery. 1993; 32:743-747.

4. Qureshi A, Sell P. Cauda Equina syndrome treated by surgical decompression: the influence of timing on surgical outcome. Eur Spine J. 2007;16(12):2143-2151.

5. MCCarthy MJH, Aylott CEW, Grevitt MP, et al. Cauda Equina syndrome: factors affecting longterm functional and sphincteric outcome. Spine. 2007; 32(2):207-216.

6. Aly TA, Aboramadan MO. Efficacy of delayed decompression of lumbar disk herniation causing cauda equina syndrome. Orthopedics. 2014 Feb;37(2):e153-6. 
